\title{
Acknowledgement to Reviewers of Vibration in 2019
}

\section{Vibration Editorial Office}

MDPI, St. Alban-Anlage 66, 4052 Basel, Switzerland

Published: 19 January 2020

The editorial team greatly appreciates the reviewers who have dedicated their considerable time and expertise to the journal's rigorous editorial process over the past 12 months, regardless of whether the papers are finally published or not. In 2019, a total of 19 papers were published in the journal, with a median time to first decision of 18 days and a median time from submission to publication of 63 days. The editors would like to express their sincere gratitude to the following reviewers for their generous contribution in 2019:

\author{
Aggogeri, Francesco \\ Aleksandrova, Mariya \\ Anghelache, Gabriel \\ Antoni, Jerome \\ Aydogdu, Metin \\ Bedon, Chiara \\ Cao, Dongxing \\ Cazzani, Antonio \\ Di Matteo, Alberto \\ Dinh, Toan \\ Dinis, Maria De Lurdes \\ Elahi, Hassan \\ Farokhi, Hamed \\ Fasel, Benedikt \\ Fiammetta, Venuti \\ Fredianelli, Luca \\ Giovannelli, Ambra \\ Gregori, Santiago \\ Ham, Suyun \\ Herbert Acero, José Francisco \\ Hirata, Yoshito \\ Hoffmann, Norbert \\ Huang, Yu-Hsi \\ Hwang, Myun Joong \\ Janusas, Giedrius \\ Kang, Jaeyoung \\ Khazaeinejad, Payam \\ Khirallah, Kareem \\ Kim, Chan-Jung \\ Kim, Soaram \\ Kong, Kian Hau \\ Koszewnik, Andrzej \\ Kuan, Yean-Der \\ Kudra, Grzegorz \\ $\mathrm{Li}, \mathrm{Li}$
}

\author{
Liang, Jun-Rui \\ Lin, Lan \\ Liu, Kefu \\ Liu, Tzong-shi \\ Loccufier, Mia \\ Madinei, Hadi \\ Mao, Yao \\ Marzband, Mousa \\ Masoodi, Amirreza \\ Massi, Francesco \\ Moore, Keegan \\ Mori, Kotaro \\ Morrey, Denise \\ Navarra, Giacomo \\ Ntotsios, Evangelos \\ Olejnik, Paweł \\ Ouakad, Hassen \\ Pajaziti, Arbnor \\ Pakrashi, Vikram \\ Papangelo, Antonio \\ Perez-Ramirez, Carlos \\ Pruncu, Catalin \\ Qian, Jin-yuan \\ Qiu, Zhicheng \\ Ricciardi, Giuseppe \\ Rustighi, Emiliano \\ Shafiee, Mahmood \\ Small, Michael \\ Smith, Ian \\ Stephen, Neil G. \\ Strano, Salvatore \\ Sun, Shih-Wei \\ Tan, Chin Yaw \\ Temel, Beytullah \\ Tiboaca, Daniela
}


Tison, Thierry

Wong, Wai

Woodhouse, Jim
Zarepoor, Masoud

Zhang, Long

(C) 2020 by the authors. Licensee MDPI, Basel, Switzerland. This article is an open access article distributed under the terms and conditions of the Creative Commons Attribution (CC BY) license (http://creativecommons.org/licenses/by/4.0/). 